\title{
Ezrin-Radixin-Moesin Binding Phosphoprotein 50 (EBP50) Suppresses the Metastasis of Breast Cancer and HeLa Cells by Inhibiting Matrix Metalloproteinase-2 Activity
}

\author{
LEI WANG ${ }^{1 *}$, YIJUN QI $^{2 *}$, YING XIONG ${ }^{2}$, ZHIQIANG PENG ${ }^{2}$, QIANG MA ${ }^{2}$, \\ YUE ZHANG ${ }^{2}$, JIAN SONG ${ }^{1}$ and JUNFANG ZHENG $^{2,3}$ \\ ${ }^{1}$ Department of Urology, Beijing Friendship Hospital, Capital Medical University, Beijing, P.R. China; \\ ${ }^{2}$ Department of Biochemistry and Molecular Biology, Capital Medical University, Beijing, P.R. China; \\ ${ }^{3}$ Beijing Key Laboratory for Tumor Invasion and Metastasis, Beijing International Cooperation \\ Base for Science and Technology on China-UK Cancer Research, Beijing, P.R. China
}

\begin{abstract}
Background: Expression of ezrin-radixin-moesinbinding phosphoprotein-50 (EBP50) is correlated with human breast and cervical cancer development, but its effects on the metastasis of breast and cervical cancer and the underlying mechanism are not fully understood. Materials and Methods: In this study, EBP50 was overexpressed in MDA-MB-231 breast cancer and HeLa cervical cancer cells; moreover, EBP50 was knocked-down in MCF-7 breast cancer cells and HeLa cells. Metastasisrelated ability and matrix metalloproteinase-2 (MMP-2) activity of these cells were investigated. Results: Cell adhesion, wound-healing and invasion were significantly suppressed in EBP50-overexpressing cells. Contrarily, EBP50-knockdown promoted cell adhesion, wound healing and invasion. EBP50 overexpression inhibited MMP-2 activity, and the knockdown of EBP50 promoted the activity of MMP-2, suggesting that EBP50 inhibited cell metastasis via suppression of MMP-2 activity. Conclusion: Our work reveals the anti-metastatic effect and a new mechanism of EBP50 action in breast and cervical cancer cells.
\end{abstract}

Breast cancer and cervical carcinoma are common tumors in females. Breast cancer is also the most common cause of cancer death among women $(522,000$ deaths in 2012) and the most frequently diagnosed cancer among women in 140

*These Authors contributed equally to this work.

Correspondence to: Department of Biochemistry and Molecular Biology, Capital Medical University, No.10 Xitoutiao, You An Men, Beijing 100069, P.R. China. Tel: +86 1083950184, e-mail: zhengjf@ccmu.edu.cn

Key Words: EBP50, adhesion, migration, matrix metalloproteinase-2. out of 184 countries worldwide. In recent years the incidence and mortality rates of breast cancer have continuously increased. Cervical cancer is the fourth most common cancer affecting women worldwide, after breast, colorectal, and lung cancer (1). Breast cancer and cervical carcinoma generally show slow growth, with symptoms occurring in advanced stages once the tumor has been discovered. Adhesion, migration and metastasis are the causes of high mortality of breast cancer and cervical cancer. Prevention of migration and metastasis of breast and cervical cancer helps improve treatment and remains a research challenge.

Ezrin-radixin-moesin-binding phosphoprotein-50 (EBP50) is a multifunctional scaffolding protein that exerts different functions in a variety of cancer types through its interactions with oncogenic or tumor-suppressive proteins (2-5). In breast cancer, EBP50 was reported to inhibit cell proliferation by regulating epidermal growth factor receptor (EGFR) or platelet-derived growth factor receptor (PDGFR) signaling and modulating the expression level of phosphatase and tensin homolog (PTEN) (3, 6-11). However, the role of EBP50 in migration and metastasis of breast cancer remains unclear. The role of EBP50 in cervical cancer metastasis has also not been reported as far as we are aware.

In this study, we sought to determine the effects of EBP50 expression on the adhesion and migration of breast cancer and cervical carcinoma cells by overexpressing EBP50 in MDA-MB-231 and HeLa cell lines. Moreover, adhesion and migration were also examined in EBP50-knockdown MCF-7 and HeLa cells.

\section{Materials and Methods}

Plasmids. The pBK-CMV-HA empty vector and pBK-CMV-HAEBP50 expression plasmid were kindly provided by Dr. Randy Hall from Emory University (Atlanta, GA, USA). pSuper.puro luciferase control shRNA and pSuper.puro EBP50 shRNA plasmids were 
kindly provided by Margaret J. Wheelock from University of Nebraska Medical Center (Omaha, NE, USA).

Cell lines and cell culture. The human breast cancer cell lines MDAMB-231, MCF-7 and human cervical cancer cell line HeLa were obtained from the American Type Culture Collection (Manassas, VA, USA). MDA-MB-231 cells were cultured in GIBCO RPMI-1640 medium (Hyclone, Logan, UT, USA). MCF-7 and HeLa cells were cultured in Dulbecco's modified Eagle's medium (DMEM; Invitrogen, Carlsbad, CA, USA). Both media were supplemented with $10 \%$ fetal bovine serum (FBS; Hyclone) and $1 \%$ antibiotic-antimycotic agent (Life Technologies, Inc, Grand Island, NY, USA). Cells were grown at $37^{\circ} \mathrm{C}$ and with $5 \% \mathrm{CO}_{2}$ to $80 \%$ confluency for use.

Establishment of cell pool in which EBP50 was stably overexpressed or knocked down. For stable overexpression of EBP50, MDA-MB231 and HeLa cells were transfected with $2 \mu \mathrm{g}$ pBK-CMV-HAEBP50 plasmid or pBK-CMV-HA vector using Lipofectamine 2000 (Invitrogen). For stable knockdown of EBP50, MCF-7 and HeLa cells were transfected with pSuper.puro-EBP50 shRNA or control pSuper.puro-luciferase shRNA plasmid respectively using Hifectin II (Applygen Technologies Inc., Beijing, P.R. China) following the manufacturer's protocol. Two days following transfection, cells were transferred to $100-\mathrm{mm}$ plates and cultured in selection medium with $350 \mu \mathrm{g} / \mathrm{ml} \mathrm{G} 418$ (Amresco, Solon, OH, USA) for cells with EBP50 overexpression and $0.5 \mu \mathrm{g} / \mathrm{ml}$ puromycin (Sigma-Aldrich, St. Louis, MO, USA) for cells with EBP50 knockdown. The medium was changed every 2 days to remove floating dead cells, and resistant colonies formed were harvested and plated in 24-well plates. Cell cultures were expanded and cultured for at least 1 month, and fractions were used for analysis of EBP50 expression by western blotting, with $\beta$-actin expression as a protein loading control. Stablytransfected cell pools were maintained and passaged in culture medium with G418 $(200 \mu \mathrm{g} / \mathrm{ml})$ or puromycin $(0.25 \mu \mathrm{g} / \mathrm{ml})$.

Western blotting. Cells were collected and lysed in radioimmunoprecipitation buffer (Beijing CoWin Biotech Co., Ltd., Beijing, PR China) in the presence of protease inhibitors for $20 \mathrm{~min}$ to extract total protein from cells stably overexpressing or with knockdown of EBP50, and protein levels were quantified using BCA assays (Beijing CoWin Biotech Co., Ltd., Beijing, PR China). Next, $50 \mu \mathrm{g}$ protein from each sample was loaded onto $8 \%$ sodium dodecyl sulfate (SDS) polyacrylamide gels and subjected to SDSpolyacrylamide gel electrophoresis (PAGE). Protein was then transferred to nitrocellulose membranes (Sigma). Membranes were blocked in blot buffer (2\% nonfat dry milk, $0.1 \%$ Tween $20,50 \mathrm{mM}$ $\mathrm{NaCl}, 10 \mathrm{mM}$ Hepes, $\mathrm{pH}$ 7.4) for $1 \mathrm{~h}$ and were then incubated with rabbit primary antibodies to human EBP50 (1:1,000 dilution; Abcam, Cambridge, UK) overnight at $4{ }^{\circ} \mathrm{C}$, followed by incubation with goat anti-rabbit horseradish peroxidase-conjugated secondary antibody (1:1,000 dilution; Abcam) for $1 \mathrm{~h}$ at room temperature. Detection was facilitated using an ECL kit (CoWin Biotech), and images were analyzed using Image J (version 1.62; National Institutes of Health, Bethesda, MD, USA).

Adhesion assay. CytoMatrix ${ }^{\mathrm{TM}}$ Cell Adhesion Strips (Millipore, Billerica, MA, USA) were used for adhesion assays. The strips were rehydrated with $200 \mu \mathrm{l}$ of PBS per well of 96-well plates for at least $15 \mathrm{~min}$ at room temperature. Cells were digested using an enzymatic dissociation approach to prepare single-cell suspensions. Cell density was also optimized by titration in serum-free medium and finally $10^{6}$ cells/well was used. One hundred microliters of the diluted cell suspension was added to each well and the plate was incubated at $37^{\circ} \mathrm{C}$ for $30 \mathrm{~min}, 60 \mathrm{~min}$ and $90 \mathrm{~min}$ in a $\mathrm{CO}_{2}$ incubator. Twenty microliters of CCK8 reagent was added to each well and the plates were incubated for $1 \mathrm{~h}$ at room temperature. The supernatant was subsequently taken to determine the absorbance value at $450 \mathrm{~nm}$ on a microplate reader. The absorbance value from bovine serum albumincoated wells was subtracted from the raw data of treatment groups.

Wound-healing assay. Cells were plated at $2 \times 10^{5}$ cells per well in 6 -well plates and grown to $100 \%$ confluency. A wound was created in the cell monolayer, and an image was immediately taken $(0 \mathrm{~h})$. Migration was then observed every $12 \mathrm{~h}$, and the closing wound was imaged. Image-Pro Plus (Media Cybernetics, Rockville, MD, USA) analysis software was used to facilitate the calculation of the relative migration rate as the relative distance recorded at $0 \mathrm{~h}$ minus that recorded at $12 \mathrm{~h}$ and $24 \mathrm{~h}$ divided by the relative distance recorded at $0 \mathrm{~h} \times 100 \%$.

Transwell assay. Cell invasion was performed using transwell inserts (Corning Costar, Cambridge, MA, USA) with polycarbonate membranes ( $8 \mu \mathrm{m}$ pore size) in 24 -well plates. For invasion assays, the filter was coated with $20 \mu \mathrm{g} / \mathrm{ml}$ Matrigel (Becton-Dickinson, San Jose, CA, USA) overnight at $4^{\circ} \mathrm{C}$. A total of $100 \mu \mathrm{l}$ cells cultured in serum-free medium $\left(1.0 \times 10^{5} / \mathrm{ml}\right)$ were seeded in the upper chamber. DMEM with $20 \%$ FBS was added to the lower chamber. After $24 \mathrm{~h}$ incubation at $37^{\circ} \mathrm{C}$ in $5 \% \mathrm{CO}_{2}$, the chamber was washed three times with $1 \times \mathrm{PBS}$. The membrane in the lower chamber was fixed in $4 \%$ paraformaldehyde and stained with crystal violet. The cells on the upper membrane that did not migrate were wiped with a cotton swab. The number of migrated cells was counted under a microscope in at least five fields.

Gelatin zymography assay. Activity of MMP-2 was assessed using a gelatin zymography assay. Cells were cultured with serum free medium for $24 \mathrm{~h}$ after seeding. Following incubation, $50 \mu \mathrm{l}$ of culture supernatant was mixed with sample buffer and resolved on a $12 \%$ SDS-PAGE under non-reducing conditions. The gel was copolymerized containing $0.5 \mathrm{mg} / \mathrm{ml}$ of gelatin (Sigma). The gel was washed twice for $30 \mathrm{~min}$ with renaturation buffer $(2.5 \%$ Triton X$100)$ at room temperature before incubation in the incubation buffer [50 mM Tris- $\mathrm{HCl}$ (pH 7.5), $200 \mathrm{mM} \mathrm{NaCl}, 10 \mathrm{mM} \mathrm{CaCl}_{2}, 1 \mu \mathrm{M}$ $\mathrm{ZnCl}_{2}$ ] at $37^{\circ} \mathrm{C}$ for $16 \mathrm{~h}$. Thereafter, the gel was stained for $2 \mathrm{~h}$ in $0.25 \%$ Coomassie brilliant blue R-250 and then de-stained. White bands were observed against a blue background after de-staining, indicating gelatinolytic activity of MMP-2.

Statistical analysis. All experiments were repeated at least three times. SPSS 11.5 software (SPSS, Inc., Chicago, IL, USA) was used to analyze the results. Independent sample $t$-tests were used and differences with $p$-values of less than 0.05 were considered statistically significant.

\section{Results}

Generation of cell pool stably overexpressing or not expressing EBP50. To study the effect of EBP50 expression on adhesion and migration of breast cancer and cervical cancer cells, we combined EBP5O gain-of-function and loss- 
A

MDA-MB-231

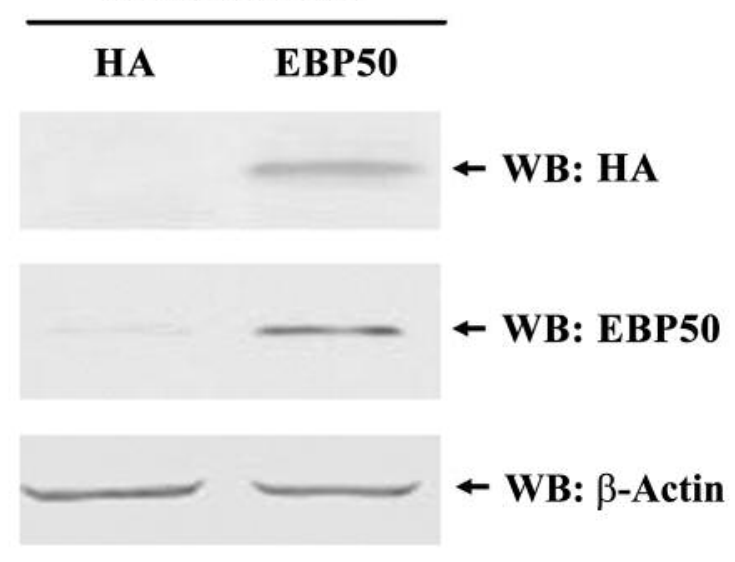

C

MCF-7

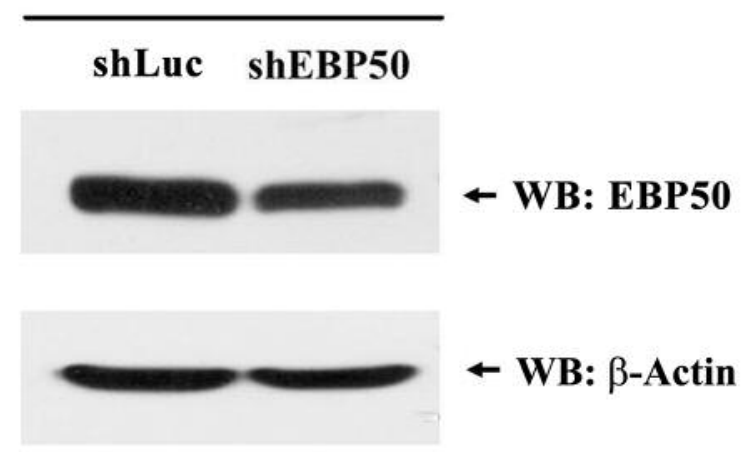

B

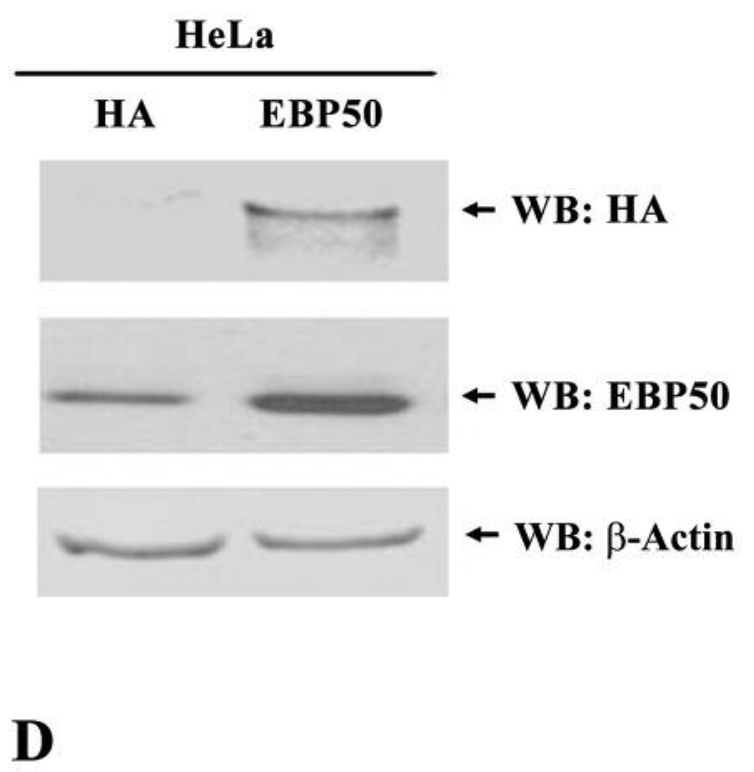

HeLa

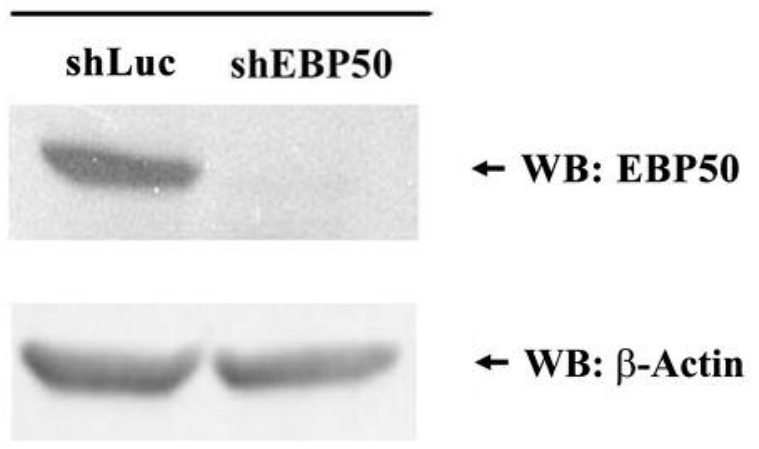

Figure 1. Expression of ezrin-radixin-moesin-binding phosphoprotein-50 (EBP50) in stably transfected breast cancer and cervical cancer cells. A: MDA-MB-231 cells. B: HeLa cells. Anti-HA and anti-EBP5O western blotting was used to detect EBP50 overexpression. In empty vector-transfected control cells (HA) cells, no HA signal and low EBP50 signal were detected. In cells stably expressing EBP50 cells, strong HA and EBP50 signal were detected. C: MCF-7 cells. D: HeLa cells. Anti-EBP50 western blotting was used to detect EBP50 knockdown. EBP50 levels in shEBP50 cells were $50 \%$ or $10 \%$ of those in shLuc control cells. $\beta$-Actin was used as a loading control.

of-function studies. Thereby MDA-MB-231 breast cancer cells, which express a low level of endogenous EBP50, were transfected with an EBP50 expression plasmid to overexpress EBP50, and MCF-7 breast cancer cells, which express a high level of endogenous EBP50, were transfected with an EBP50 shRNA plasmid to knockdown its expression. For cervical cancer cells, we chose HeLa cell to perform both EBP50 gain-of-function and loss-of-function studies. These were carried out for the purpose of observing the effect of EBP5O expression on breast cancer and cervical cancer cells.
The cell pools stably overexpressing EBP50, namely MDAMB-231-HA-EBP50 (EBP-231) and its control MDA-MB231-HA (HA-231), HeLa-HA-EBP50 (EBP-HeLa) and its control HeLa-HA (HA-HeLa), were generated by transfection with the neo-pBK-CMV-HA-EBP50 or neo-pBK-CMV-HA vector, respectively. Protein expression in these stable cells was verified by western blot analysis as shown in Figure 1A and B. HA-tagged EBP50 protein expression was not detected in control cells. In EBP-231 and EBP-HeLa cells, exogenous HA-tagged EBP50 was robustly overexpressed. 


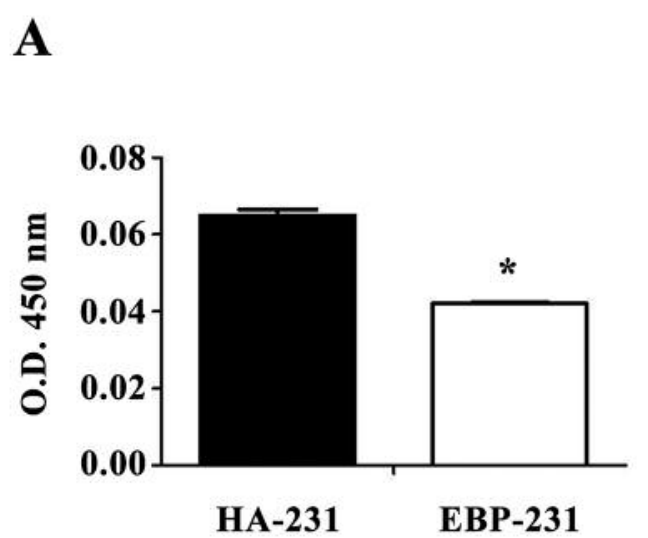

C

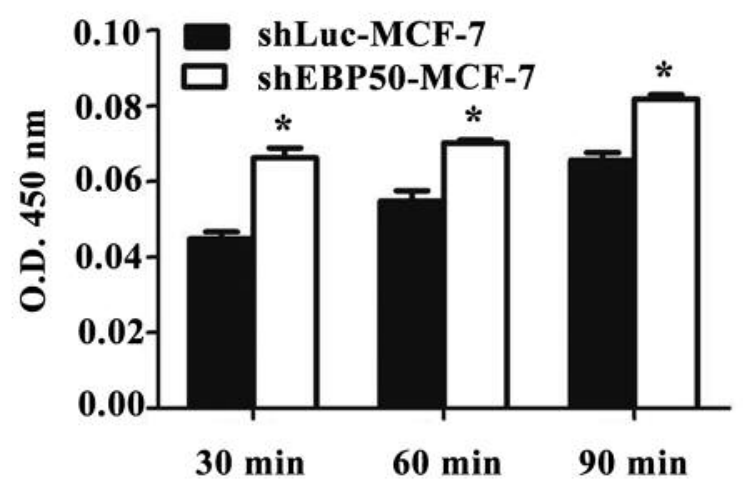

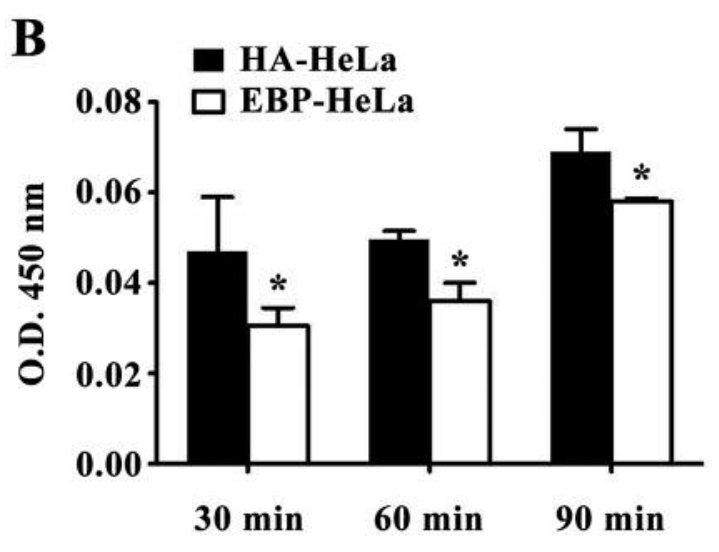

D

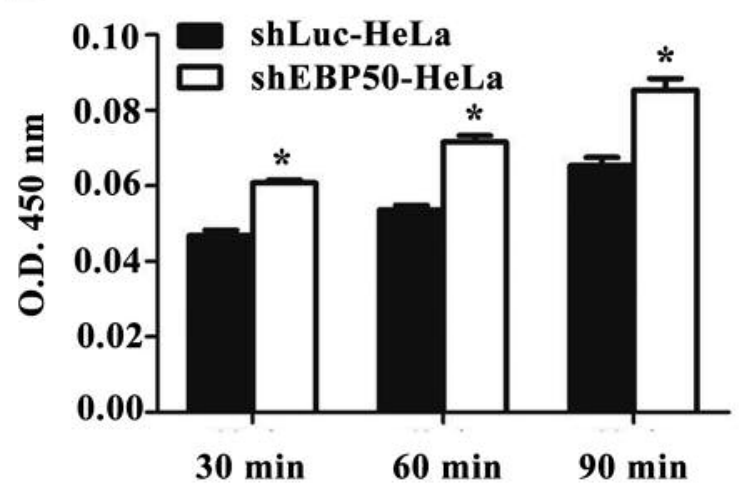

Figure 2. The anti-cell adhesion effects of ezrin-radixin-moesin-binding phosphoprotein-50 (EBP50) in breast cancer and cervical cancer cells. Adhesion assay was performed to analyze the ability of adherence to fibronectin at different time points. A: MDA-MB-231 cells. B: HeLa cells. Cell adhesion to fibronectin were inhibited in cells stably overexpressing EBP50 compared with empty vector-transfected control (HA) cells. C: MCF-7 cells. D: HeLa cells. EBP50 knockdown (shEBP50) in cells significantly promoted cell adhesion to fibronectin compared to shLuc control cells. *Significantly different from the control at $p<0.05$.

The cell pools with stable knockdown of EBP50 (shEBP50-MCF-7, shEBP50-HeLa) and their control cell lines (shLuc-MCF-7, shLuc-HeLa) were generated by transfection with the pSuper.puro EBP50 shRNA plasmid or the control pSuper.puro luciferase shRNA plasmid, respectively. Verification of protein knockdown was determined by western blot analysis as shown in Figure 1C and D. In shEBP50 transfected MCF-7 and HeLa cells, EBP50 expression was stably knocked-down by $50 \%$ and $90 \%$ compared to control shLuc-transfected cells.

EBP5O inhibits the adhesion of breast cancer and HeLa cells. Cell matrix interaction and cell motility are important for cancer cell metastasis. In order to examine the potential anti-metastatic effects of EBP50, cell matrix interaction assays were performed on MDA-MB-231, MCF-7 and HeLa cells. The results indicated that cell matrix interaction was inhibited by $E B P 50$ at multiple time points. At $60 \mathrm{~min}$, the number of MDA-MB-231 cells adhering to fibronectin was reduced by $35.4 \%$ following overexpression of EBP5O $(p<0.05$, Figure 2A). At 30, 60 and $90 \mathrm{~min}$, the number of HeLa cells adhering to fibronectin was reduced by $35.1 \%$, $27.3 \%$ and $16 \%$, respectively following overexpression of EBP50 ( $p<0.05$, Figure 2B). Conversely, EBP50 knockdown in MCF-7 and HeLa cells significantly promoted cell adhesion to fibronectin at all study time points (Figure $2 \mathrm{C}$ and D). These results suggest that expression of EBP5O significantly inhibited the adhesive abilities of MDA-MB231, MCF-7 and HeLa cells compared with those of control cells $(p<0.05)$.

EBP50 inhibits migration and invasion of breast cancer and HeLa cells. Adhesion assays indicated that EBP50 had the ability to inhibit tumor cell adherence to the matrix. The formation of focal adhesions is important in cell signaling to 
A

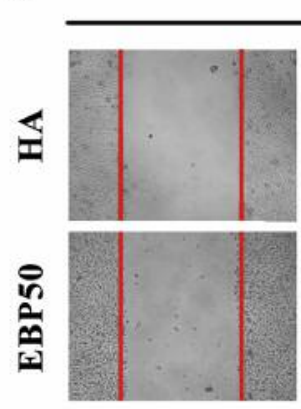

O h

B

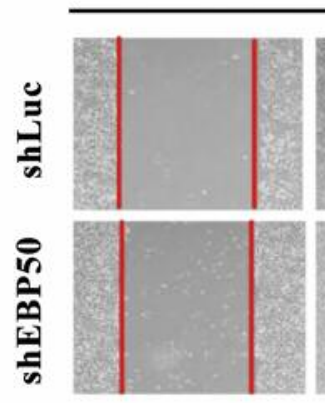

O h

C
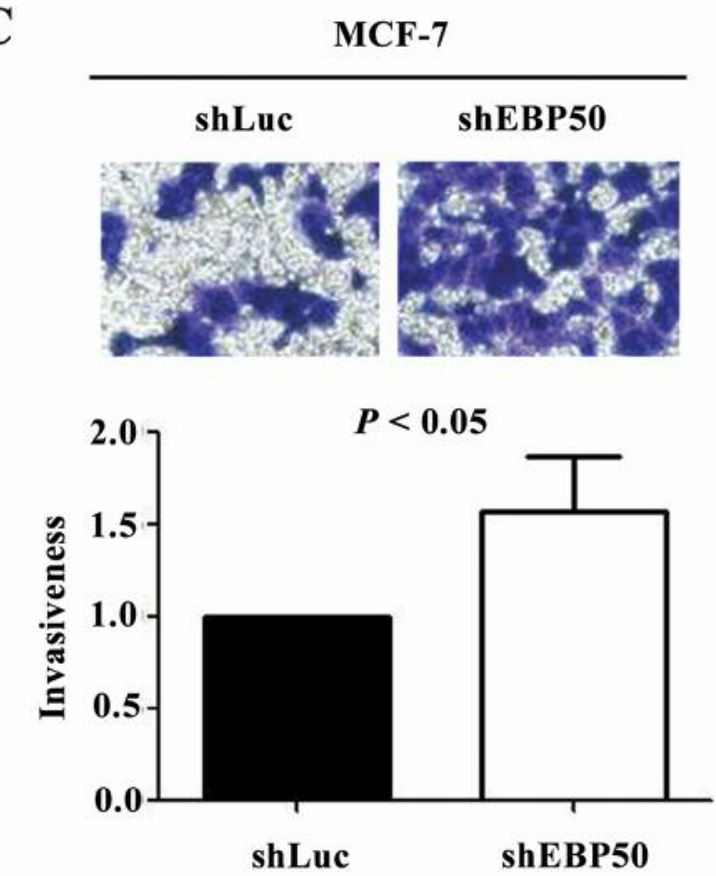

MDA-MB-231
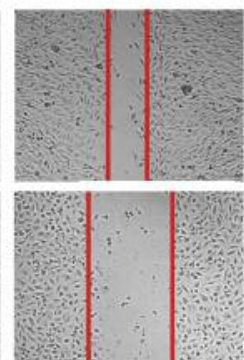

$12 \mathrm{~h}$

$24 \mathrm{~h}$

MCF-7

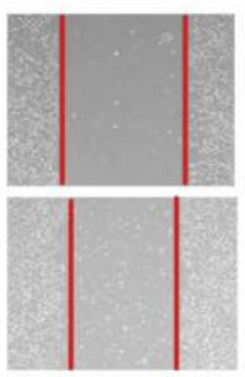

$24 \mathrm{~h}$

$12 \mathrm{~h}$

ShEBP50
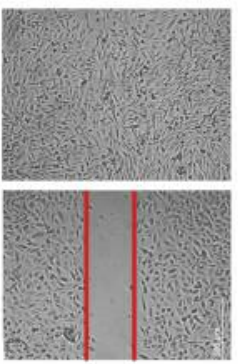

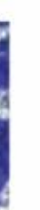


$\mathbf{A}$

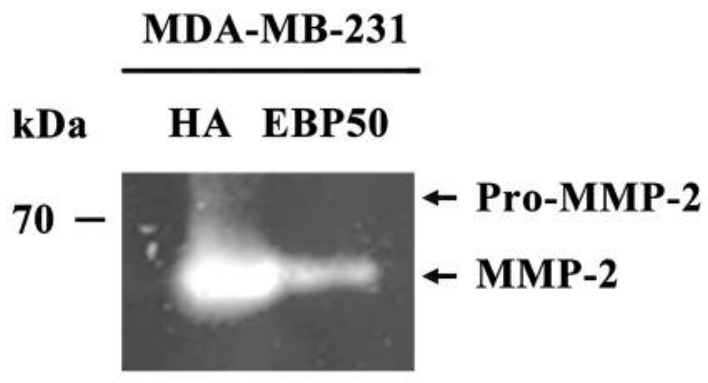

B

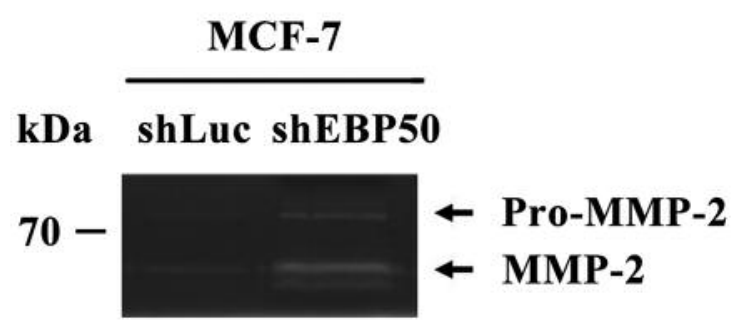

\section{HeLa}

kDa HA EBP50

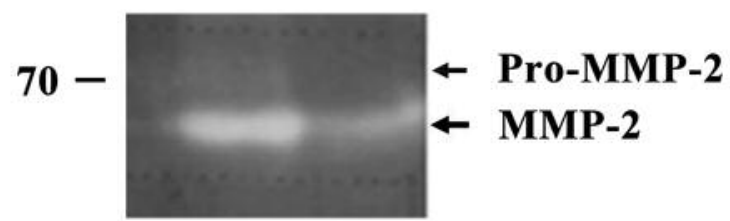

Figure 4. Inhibitory effects of ezrin-radixin-moesin-binding phosphoprotein-50 (EBP50) on matrix metalloproteinase-2 (MMP-2) activity in breast cancer and cervical cancer cells. The cells were treated with serum-free media for $24 \mathrm{~h}$, and conditioned media were collected for gelatin zymography. A: MMP-2 activity was inhibited by EBP50 overexpression in MDA-MB-231 and HeLa cells. HA: Control cells, EBP50: EBP50transfected cells. B: MMP-2 activity was promoted in EBP50 knockdown (shEBP50) in MCF-7 and HeLa cells compared with control (shLuc) cells. Data are representative of three independent experiments.

direct cell migration (12). We further investigated whether EBP50 affects cell migratory ability. At $0 \mathrm{~h}$, the same wounds were produced in two groups of cells. After $12 \mathrm{~h}$ and $24 \mathrm{~h}$, the wound width was significantly reduced in the control group, while width reduction was significantly inhibited in the EBP50 overexpressing groups (Figure $3 \mathrm{~A}, p<0.05$ ). On the contrary, EBP50 knockdown in MCF-7 and HeLa cells significantly promoted cell migration and wound closure after $12 \mathrm{~h}$ and $24 \mathrm{~h}$ (Figure 3B, $p<0.05$ ). In agreement with results from the wound-healing assay, the number of invading cells was also increased following EBP50 knockdown in an in vitro Matrigel invasion assay (Figure $3 \mathrm{C}, p<0.05$ ). These results suggest that EBP50 is able to inhibit the migration and invasion of breast cancer and cervical cancer cells.

EBP50 reduces MMP-2 activity of breast cancer and HeLa cells. Tumor cell metastasis requires degradation of the matrix. MMP-2 is one of the main proteolytic enzymes and degrades the environmental extracellular matrix and the basement membrane. In order to clarify whether the activity of MMP-2 is involved in the inhibition of migration and invasion by
EBP50 in MDA-MB-231, MCF-7 and HeLa cells, gelatin zymographic assays were performed. MDA-MB-231, MCF-7 and HeLa cells were incubated in serum-free media for $24 \mathrm{~h}$, and the conditioned media were collected to analyze activity of MMP-2. The results show that EBP50 overexpression reduced the activity of MMP-2 and EBP50 knockdown enhanced the activity of MMP-2 ( $p<0.05$; Figure 4$)$.

\section{Discussion}

In this study, our data demonstrated that EBP5O overexpression inhibited the adhesion and migration of MDAMB-231 and HeLa cells, and suppressed MMP-2 activity in these cells, while EBP50 knockdown in MCF-7 and HeLa cells promoted adhesion and migration, and enhanced the activity of MMP-2. Therefore, the results of both gain-offunction and loss-of-function assays revealed that EBP50 may represent a metastasis-suppressing protein in breast cancer and cervical carcinoma. Notably, to our knowledge, the finding that EBP50 can inhibit the metastasis-related phenotype of cervical cancer cells is novel. 
The expression level of EBP50 in breast cancer tissues varies depending on the pathological grade and cancer stage (7). In MDA-MB-231 breast cancer cells, EBP50 exhibits a relatively low endogenous expression, and we increased EBP50 expression through stable transfection with a pBKCMV-HA-EBP50 plasmid. MCF-7 breast cancer cells express relatively a high level of EBP50 and an EBP50 shRNA plasmid was used to reduce EBP50 expression. Our analysis indicated that EBP5O overexpression inhibited the adhesion and migration of MDA-MB-231 and HeLa cells, while EBP50 knockdown promoted adhesion and migration of MCF-7 and HeLa cells. Cell adhesion and migration are associated with tumor development and are the main causes of death in patients with tumors (13-15). Our results have important significance in breast and cervical cancer development and therapeutics.

Metastasis is responsible for more than $90 \%$ of cancerrelated mortality (16). A critical step in tumor metastasis is the degradation of the basement membrane, which is catalyzed by proteolytic enzymes, such as MMPs (17). Significant down-regulation of MMP-2 activity was one of the mechanisms for the EBP50-induced inhibition of migration of MDA-MB-231 and HeLa cells. Results of EBP50 knockdown further revealed MMP-2 activity played an important role in migration and invasion of MCF-7 and HeLa cells. It was also a novel observation that EBP50 regulates MMP-2 activity to modulate breast cancer and HeLa cell migration and invasion.

EBP50 was found to directly or indirectly affect cancer behavior through interaction with partners and the regulation of their signaling pathways $(11,18)$. For example, EBP50 was reported to form a protein complex with EGFR and neurofibromin $2(\mathrm{NF} 2)$ at intercellular adherens junctions (19), thereby mediating internalization and signal transduction of EGFR to regulate oncogenic processes. EBP50 was also found to interact with PDGFR to form a transduction complex with PTEN and NF2 $(3,8$, 18). This complex functioned to block the signaling associated with PDGFR, thereby affecting apoptosis during epithelial-to-mesenchymal transition and suppressing cancer development and metastasis (20). The results of this study have identified a new mechanism by which EBP5O affects cancer cell behavior.

In addition, EBP50 was reported to be an actin cytoskeleton- and cell junction-related protein $(21,22)$. Abnormal expression and organization of actin cytoskeleton and cell junctions are closely related to tumor cell metastasis. The metastasis-suppressing ability of EBP50 possibly results from its regulation of actin cytoskeleton and cell junction proteins. EBP50 expression might also change its interaction with partners which have important roles in adhesion and migration to affect breast and cervical cancer cell metastasis. These need to be further investigated.
In summary, our results demonstrate that EBP50 regulates metastasis-related behavior (adhesion and migration abilities) of MDA-MB-231, MCF-7 and HeLa cells and that EBP50 acted as a tumor metastasis-suppressing protein in breast cancer and cervical carcinoma cells. EBP50 may be a potential therapeutic target in the treatment of both cancer types.

\section{Conflicts of Interest}

The Authors declare no conflict of interest in regard to this study. The founding sponsors had no role in the design of the study; in the collection, analyses, or interpretation of data; in the writing of the manuscript, and in the decision to publish the results.

\section{Acknowledgements}

This work was supported by the National Natural Science Foundation of the People's Republic of China (No. 81372739, $81672521)$, the Importation and Development of High-Caliber Talents Project of Beijing Municipal Institutions (No. CIT\&TCD201304187), Basic-Clinic Cooperation Project of Capital Medical University (No. 14JL-L03), Scientific research fund of Beijing Friendship Hospital (No. yyqdkt2015-14).

\section{References}

1 Ferlay J, Shin H, Bray F, Forman D, Mathers C and Parkin D: Cancer Incidence and Mortality Worldwide: IARC Cancer Base No. 10. International Agency for Research on Cancer, 2010.

2 Bartholow TL, Becich MJ, Chandran UR and Parwani AV: Immunohistochemical analysis of ezrin-radixin-moesin-binding phosphoprotein 50 in prostatic adenocarcinoma. BMC Urol 11: 12, 2011.

3 Cheng S, Li Y, Yang Y, Feng D, Yang L, Ma Q, Zheng S, Meng R, Wang S, Wang S, Jiang WG and He J: Breast cancer-derived K172N, D301V mutations abolish $\mathrm{Na}^{+} / \mathrm{H}^{+}$exchanger regulatory factor 1 inhibition of platelet-derived growth factor receptor signaling. FEBS Lett 587: 3289-3295, 2013.

4 Molina JR, Morales FC, Hayashi Y, Aldape KD and Georgescu MM: Loss of PTEN-binding adapter protein NHERF1 from plasma membrane in glioblastoma contributes to PTEN inactivation. Cancer Res 70: 6697-6703, 2010.

5 Wang L, Du YR, Ji MY, Wang W, Zhan N, Zhou QS and Dong WG: Reduced EBP50 expression or mis-localization of the EBP50 protein is associated with the malignant progression of esophageal squamous cell carcinoma. Eur Rev Med Pharmacol Sci 18: 3854-3863, 2014.

6 Bellizzi A, Greco MR, Rubino R, Paradiso A, Forciniti S, Zeeberg K, Cardone RA and Reshkin SJ: The scaffolding protein NHERF1 sensitizes EGFR-dependent tumor growth, motility and invadopodia function to gefitinib treatment in breast cancer cells. Int J Oncol 46: 1214-1224, 2015.

7 Pan Y, Wang L and Dai JL: Suppression of breast cancer cell growth by $\mathrm{Na}^{+} / \mathrm{H}^{+}$exchanger regulatory factor 1 (NHERF1). Breast Cancer Res 8: R63, 2006.

8 Vaquero J, Nguyen Ho-Bouldoires TH, Claperon A and Fouassier L: Role of the PDZ-scaffold protein NHERF1/EBP50 in cancer biology: from signaling regulation to clinical relevance. Oncogene 36: 3067-3079, 2017. 
9 Yang L, Wang Y, Chen P, Hu J, Xiong Y, Feng D, Liu H, Zhang $\mathrm{H}$, Yang $\mathrm{H}$ and $\mathrm{He} \mathrm{J}: \mathrm{Na}(+) / \mathrm{H}(+)$ exchanger regulatory factor 1 (NHERF1) is required for the estradiol-dependent increase of phosphatase and tensin homolog (PTEN) protein expression. Endocrinology 152: 4537-4549, 2011.

10 Yao W, Feng D, Bian W, Yang L, Li Y, Yang Z, Xiong Y, Zheng $\mathrm{J}$, Zhai R and He J: EBP50 inhibits EGF-induced breast cancer cell proliferation by blocking EGFR phosphorylation. Amino Acids 43: 2027-2035, 2012.

11 Zheng JF, Sun LC, Liu H, Huang Y, Li Y and He J: EBP50 exerts tumor suppressor activity by promoting cell apoptosis and retarding extracellular signal-regulated kinase activity. Amino Acids 38: 1261-1268, 2010.

12 Jacquemet G, Hamidi $\mathrm{H}$ and Ivaska J: Filopodia in cell adhesion, 3D migration and cancer cell invasion. Curr Opin Cell Biol 36: 23-31, 2015.

13 Halon A and Donizy P: The role of the ERM protein family in maintaining cellular polarity, adhesion and regulation of cell motility. Postepy Hig Med Dosw 66: 158-164, 2012 (in Polish).

14 Kreimann EL, Morales FC, de Orbeta-Cruz J, Takahashi Y, Adams H, Liu TJ, McCrea PD and Georgescu MM: Cortical stabilization of beta-catenin contributes to NHERF1/EBP50 tumor suppressor function. Oncogene 26: 5290-5299, 2007.

15 Zheng J, Dai Y, Yang Z, Yang L, Peng Z, Meng R, Xiong Y and He J: Ezrin-radixin-moesin-binding phosphoprotein-50 regulates EGF-induced AKT activation through interaction with EGFR and PTEN. Oncol Rep 35: 530-537, 2016.

16 Yu S, Yang CS, Li J, You W, Chen J, Cao Y, Dong Z and Qiao Y: Cancer prevention research in China. Cancer Prev Res 8: 662674, 2015.

17 Wells A, Grahovac J, Wheeler S, Ma B and Lauffenburger D: Targeting tumor cell motility as a strategy against invasion and metastasis. Trends Pharmacol Sci 34: 283-289, 2013.
18 Ma L, Young J, Prabhala H, Pan E, Mestdagh P, Muth D, Teruya-Feldstein J, Reinhardt F, Onder TT, Valastyan S, Westermann F, Speleman F, Vandesompele J and Weinberg RA: miR-9, a MYC/MYCN-activated microRNA, regulates Ecadherin and cancer metastasis. Nat Cell Biol 12: 247-256, 2010.

19 Paoletti C and Hayes DF: Molecular testing in breast cancer. Annu Rev Med 65: 95-110, 2014.

20 Isaacson KJ, Martin Jensen M, Subrahmanyam NB and Ghandehari $\mathrm{H}$ : Matrix-metalloproteinases as targets for controlled delivery in cancer: An analysis of up-regulation and expression. J Control Release, 2017. https://doi.org/10. 1016/j.jconrel.2017.01.034. [Epub ahead of print]

21 Chiasson-MacKenzie C, Morris ZS, Baca Q, Morris B, Coker JK, Mirchev R, Jensen AE, Carey T, Stott SL, Golan DE and McClatchey AI: NF2/Merlin mediates contact-dependent inhibition of EGFR mobility and internalization via cortical actomyosin. J Cell Biol 211: 391-405, 2015.

22 Malavaki CJ, Roussidis AE, Gialeli C, Kletsas D, Tsegenidis T, Theocharis AD, Tzanakakis GN and Karamanos NK: Imatinib as a key inhibitor of the platelet-derived growth factor receptor mediated expression of cell surface heparan sulfate proteoglycans and functional properties of breast cancer cells. FEBS J 280: 2477-2489, 2013. 